\title{
The Usefulness of Immunocytochemistry of CD56 in Determining Malignancy from Indeterminate Thyroid Fine-Needle Aspiration Cytology
}

\author{
Hyunseo Cha $\cdot$ Ju Yeon Pyo \\ Soon Won Hong \\ Department of Pathology, Gangnam Severance \\ Hospital, Seoul, Korea
}

\begin{abstract}
Background: Fine-needle aspiration cytology serves as a safe, economical tool in evaluating thyroid nodules. However, about $30 \%$ of the samples are categorized as indeterminate. Hence, many immunocytochemistry markers have been studied, but there has not been a single outstanding marker. We studied the efficacy of CD56 with human bone marrow endothelial cell marker-1 (HBME-1) in diagnosis in the Bethesda System for Reporting Thyroid Cytopathology (TBSRTC) category III. Methods: We reviewed ThinPrep liquid-based cytology (LBC) samples with Papanicolaou stain from July 1 to December 31, 2016 (2,195 cases) and selected TBSRTC category III cases $(n=363)$. Twenty-six cases were histologically confirmed as benign (six cases, $23 \%$ ) or malignant (20 cases, $77 \%$ ); we stained 26 LBC slides with HBME-1 and CD56 through the cell transfer method. For evaluation of reactivity of immunocytochemistry, we chose atypical follicular cell clusters. Results: CD56 was not reactive in 18 of 20 cases (90\%) of malignant nodules and showed cytoplasmic positivity in five of six cases (83\%) of benign nodules. CD56 showed high sensitivity $(90.0 \%)$ and relatively low specificity $(83.3 \%)$ in detecting malignancy $(p=.004)$. HBME-1 was reactive in 17 of 20 cases (85\%) of malignant nodules and was not reactive in five of six cases (83\%) of benign nodules. HBME-1 showed slightly lower sensitivity (85.0\%) than CD56. The specificity in detecting malignancy by HBME-1 was similar to that of CD56 (83.3\%, $p=.008$ ). CD56 and HBME- 1 tests combined showed lower sensitivity $(75.0 \%$ vs $90 \%)$ and higher specificity (93.8\% vs $83.3 \%)$ in detecting malignancy compared to using CD56 alone. Conclusions: Using CD56 alone showed relatively low specificity despite high sensitivity for detecting malignancy. Combining CD56 with HBME-1 could increase the specificity. Thus, we suggest that CD56 could

be a useful preoperative marker for differential diagnosis of TBSRTC category III samples.
\end{abstract}

Key Words: Biopsy, fine-needle; Thyroid fine-needle aspiration; Immunohistochemical staining

CD56; HBME-1

\author{
Received: July 6, 2018 \\ Acced: September 13, 2018

\section{Corresponding Author} \\ Soon Won Hong, MD \\ Department of Pathology, Gangnam Severance \\ Hospital, 211 Eonju-ro, Gangnam-gu, Seoul 06273, \\ Korea \\ Tel: +82-2-2019-3540 \\ Fax: $+82-2-3463-2103$ \\ E-mail: SOONWONH@yuhs.ac
}

Thyroid nodules, composed of non-neoplastic and neoplastic lesions, are found in the general population at a rate of about $5 \% .^{1}$ In Korea, as of 2011, the diagnosis of thyroid carcinoma has increased as much as 15 times compared to $1993 .{ }^{2}$ One of the reasons for this increase is thought to be from development of the fine-needle aspiration cytology (FNAC) technique, which is fast and accurate. FNAC plays a crucial role in treating thyroid carcinoma, such as in predicting a malignant nodule or in helping physicians make reasonable choices between surgery and safe follow-up treatment. ${ }^{3}$ For all the benefits of FNAC, the cytopathology reports are often either ambiguous or difficult to interpret. The words "atypical," "indeterminate," or "cannot be excluded" may cause confusion in patient management and diagnosis. ${ }^{4}$ The Bethesda System for Reporting Thyroid Cytopathology (TBSRTC) was developed to report FNA and to provide a unified terminology and diagnostic criteria for associating these cyto- logical diagnoses with clinical management. ${ }^{5,6}$

Papillary thyroid carcinoma (PTC) is the most common malignant lesion representing $70 \%-85 \%$ of all thyroid cancer and is usually diagnosed by its morphologic features such as papillary structures, ground glass nuclei, nuclear grooves, and nuclear inclusions. ${ }^{7-16}$ But, when a sample has a small amount of follicular cells, it is too difficult to make a correct diagnosis. ${ }^{17-19}$ Hence, many studies have advocated the use of immunocytochemistry markers and ancillary techniques that use a molecular panel in the purifying process. ${ }^{20-30}$ Many types of single or multiple panels of immunohistochemical markers were studied to determine the optimal marker of malignancy; human bone marrow endothelial cell marker-1 (HBME-1), galectine-3, and cytokeratin-19 were shown to have high diagnostic accuracy. ${ }^{24-36}$ We studied the application of CD56 immunocytochemistry with liquid-based cytology (LBC) for samples that had been diagnosed as TBSRTC category 
III. Additionally, we evaluated the efficacy of the marker CD56 with HBME-1.

\section{MATERIALS AND METHODS}

\section{Patients and samples}

We conducted a prospective study from July 2016 to December 2016 and archived 2,195 Papanicolaou (Pap)-stained slides retrieved from the pathology department. Each author had access to the patient profiles. The thyroid nodules were examined initially by ultrasonography; the size varied from 4 to $25 \mathrm{~mm}$. Cytologic cases of the baseline period were classified according to the TBSRTC classification. The cytology results were distributed accordingly: TBSRTC I, 8.3\%; TBSRTC II, 28\%; TBSRTC III, 16.5\%; TBSRTC IV, $1 \%$; TBSRTC V, $12.5 \%$; and TBSRTC VI, $33.7 \%$. The aim of our study was to evaluate the diagnostic value of CD56 in indeterminate cytology cases. All cases that belonged to TBSRTC category III $(\mathrm{n}=363)$ were studied. All cases were handled through the LBC method and with the help of a ThinPrep 5000 processor (Hologic Co., Marlborough, MA, USA). The LBC slides were fixed using methanol and later stained with Pap. Leftover materials were stored using PreservCyt for possible future studies, including immunocytochemistry. Twenty-six cases were histologically confirmed as either benign (6 cases, 23\%) or malignant (20 cases, 77\%); we stained 26 LBC slides with HBME-1 and CD56 through the cell transfer method.

\section{Cell transfer and immunocytochemistry}

The Pap-stained slide of FNAC and the area of the smeared atypical cell were marked by a pathologist. Atypical clusters could be selected for staining from each LBC slide by the cell transfer method. The previously described cell delivery technique was performed at the marked spot on the slide. ${ }^{37-39}$ The cover slip was separated from the Pap-stained smear slide, overlaid with Malinol (Muto Chemical, Tokyo, Japan), and heated overnight at $70^{\circ} \mathrm{C}-80^{\circ} \mathrm{C}$. They were then incubated for 1 hour in a warm container at $50^{\circ} \mathrm{C}-60^{\circ} \mathrm{C}$ to lighten the Malinol films. We stripped the Malinol film containing the cells from the slide and cut the marked spots covered in the Malinol film into pieces concordant to the evident spot from the primary slide. The Malinol film was moved to another glass slide, incubated at $70^{\circ} \mathrm{C}$ for about 2 hours, and removed using xylene. ${ }^{37-39}$

Immunocytochemistry uses the following immune staining markers: HBME-1 (1:100, Dako, Glustrup, Denmark) and CD56 (1:100, Ventana, Tucson, AZ, USA). Positive immuno- histochemical staining showed moderate or more cytoplasmic positivity for at least $30 \%$ of epithelial-follicular cells in all cytological cases. Histological diagnosis and a $30 \%$ immunocytochemistry cutoff were applied to reduce false-positive or falsenegative outcomes. ${ }^{34,35}$

We did not distinguish between moderately positive or strongly positive in levels of immunostaining, and designated both moderate positive and strongly positive as benign in whole. While CD56 stained the cytoplasm, HBME-1 stained the cytoplasm and membrane. We identified mesothelial cells as the positive control with HBME-1 and histiocytes/macrophages for CD56 positive control. We identified lymphocytes as the negative control. We compared with paraffin blocks for immunohistochemistry. Immunohistochemistry analysis did not reveal cellto-tissue mismatch yields; both cytology and specific histologic samples were coincident. We used buffered formaldehyde to fix the surgical samples. The paraffin blocks were cut into 5 - $\mu$ m-thick sections and stained with hematoxylin-eosin. All fibroadipose tissues that were adjacent to the thyroid were extensively searched to find lymph nodes.

We sought true papillary structure with nuclear characteristics to detect PTC and diagnosed follicular variant papillary thyroid carcinoma (FVPTC) when there were characteristics matching PTC in multiple sites.

\section{Statistical analysis}

The statistical data were analyzed using SPSS software ver. 23.0 (IBM Corp., Armonk, NY, USA) and Fisher exact test; pvalues less than .05 were acknowledged as statistically significant.

All procedures performed in the current study were approved by institutional review board (IRB) in Gangnam Severance Hospital (local IRB number: 3-2018-0096, May 21, 2018) in accordance with the 1964 Helsinki declaration and its later amendments. Formal written informed consent was not required with a waiver by the appropriate IRB.

\section{RESULTS}

As emphasized earlier in the materials and methods section, during our study period from July 2016 to December 2016, we analyzed 2,195 samples from thyroid FNAC and selected 363 samples of TBSRTC category III using an immunocytochemistry panel composed of HBME-1 and CD56 (Fig. 1). Among 353 cases of indeterminate thyroid nodules with category III, 26 patients who had been surgically treated were selected. Three male and 23 female patients were included; the median age was 


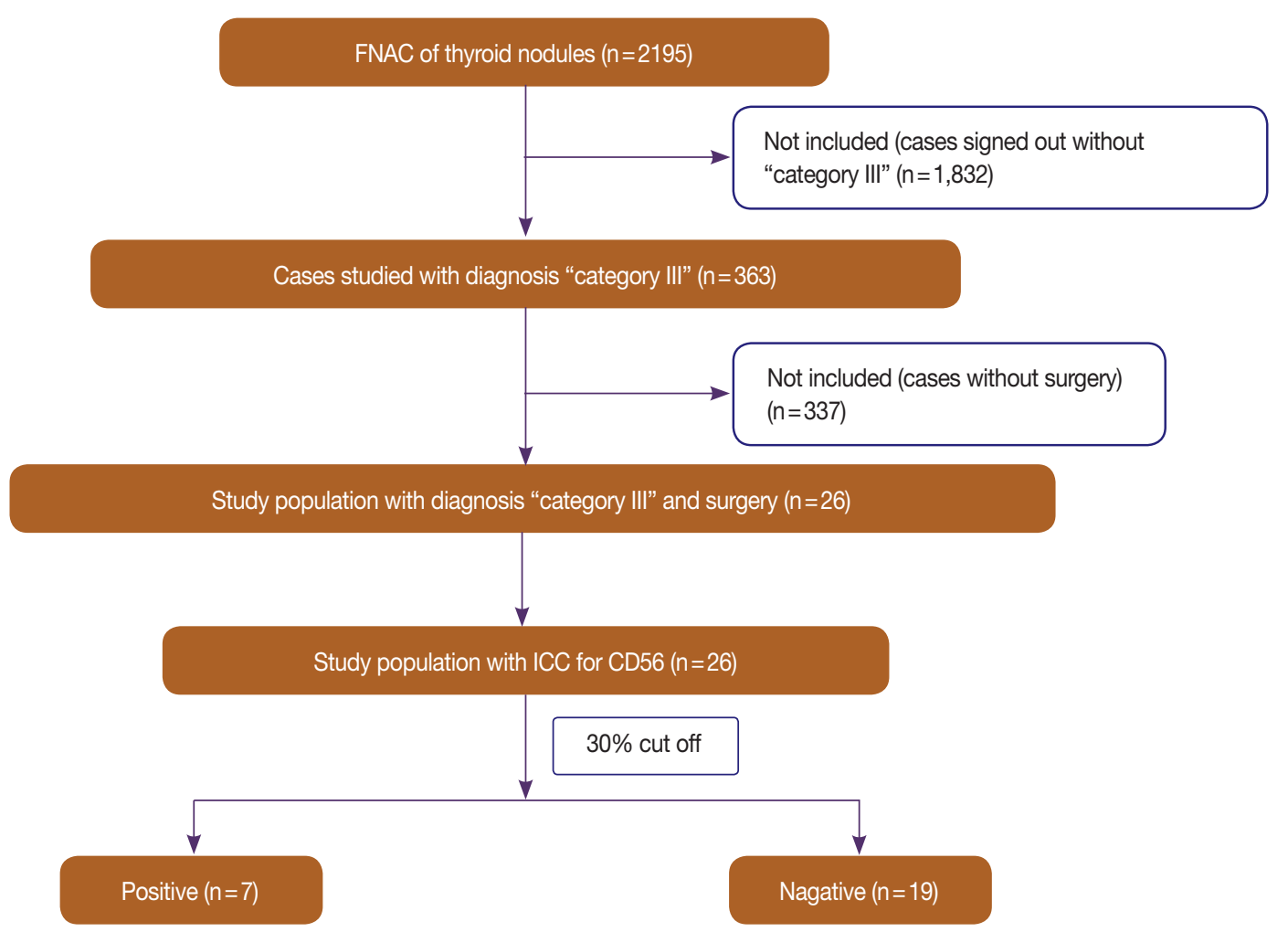

Fig. 1. Flow diagram of the study population. FNAC, fine-needle aspiration cytology; ICC, immunocytochemistry.

50 years. The surgically acquired category III samples were histologically confirmed as 10 non-malignant nodules and five adenomatous hyperplasia. Twenty nodules were malignant and 10 were conventional type PTC. Five cases were FVPTC. One case was diffuse sclerosing variant papillary thyroid carcinoma. Fig. 2 depicts the characteristics of the patients and their clinical and pathological features. We considered all FVPTC as infiltrative FVPTC.

Table 1 shows how immunostaining is expressed in two categories. In 18 of 20 cases (90\%), the malignant nodules were completely negative to CD56 (Fig. 3A, B), and two cases of FVPTC showed focal weak positivity (5\%). In contrast, five of six cases of benign nodules (83\%) stained with CD56 showed cytoplasmic and membranous positivity (Fig. 4A, B). The sensitivity was 90\% and specificity was $83.3 \%$ with diagnostic accuracy of $88.4 \%$. The CD56 results were statistically meaningful $(p=.004)$. HBME-1 was positive in 17 of 20 cases with $85 \%$ sensitivity and $83.3 \%$ specificity and diagnostic accuracy of $84 \%(\mathrm{p}=.008)$. HBME-1 showed slightly lower sensitivity (85.0\%) than that of CD56. The specificity in detecting malignancy by HBME-1 was similar to that of $\operatorname{CD} 56(83.3 \%, \mathrm{p}=.008)$.

We analyzed the outcome using both CD56 and HBME-1 (Table 2). Combined CD56 and HBME-1 tests showed lower

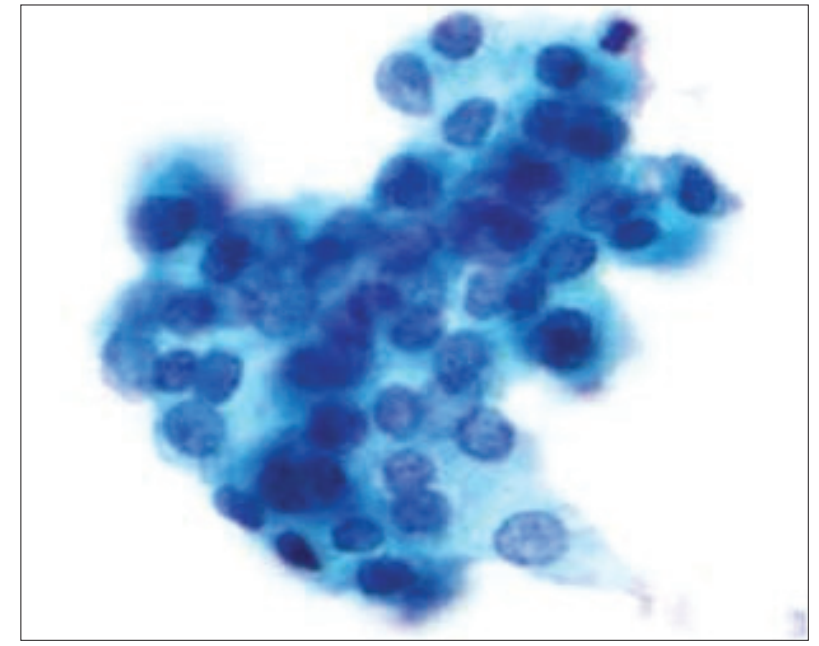

Fig. 2. A thyroid lesion diagnosed as category III on liquid-based cytology (Papanicolaou, ×400).

Table 1. CD56 and HBME-1 staining scores in the six benign nodules and 20 malignant nodules with histological follow-up

\begin{tabular}{lcrrrr}
\hline & \multicolumn{2}{c}{ CD56 } & & \multicolumn{2}{c}{ HBME-1 } \\
\cline { 2 - 3 } \cline { 5 - 6 } \cline { 5 - 6 } & Positive & Negative & & Positive & Negative \\
\hline Benign $(n=6)$ & $5(83)$ & $1(17)$ & & $1(17)$ & $5(83)$ \\
Malignant $(n=20)$ & $2(10)$ & $18(90)$ & & $17(85)$ & $3(15)$ \\
\hline
\end{tabular}

Values are presented as number (\%).

HBME-1, human bone marrow endothelial cell marker-1. 

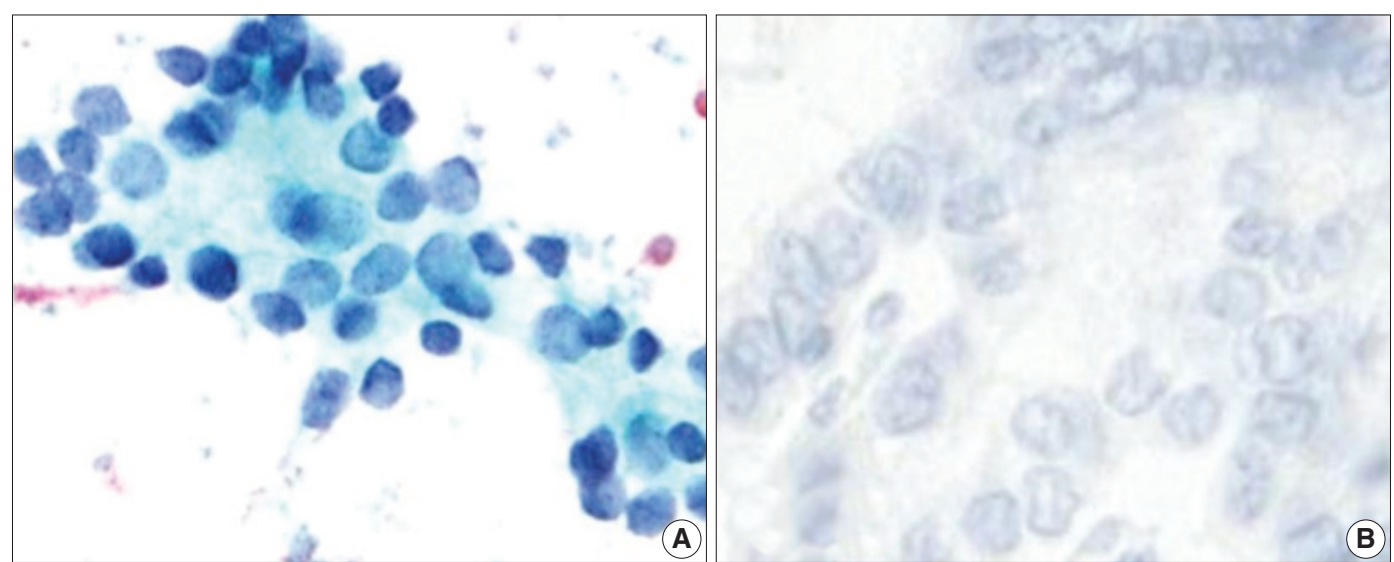

Fig. 3. (A) CD56 negativity in the case in Fig. 1 (avidin-biotin-peroxidase complex, $\times 400$ ). (B) Negative CD56 expression on the histochemical sample for the same case (avidin-biotin-peroxidase complex, $\times 400$ ).
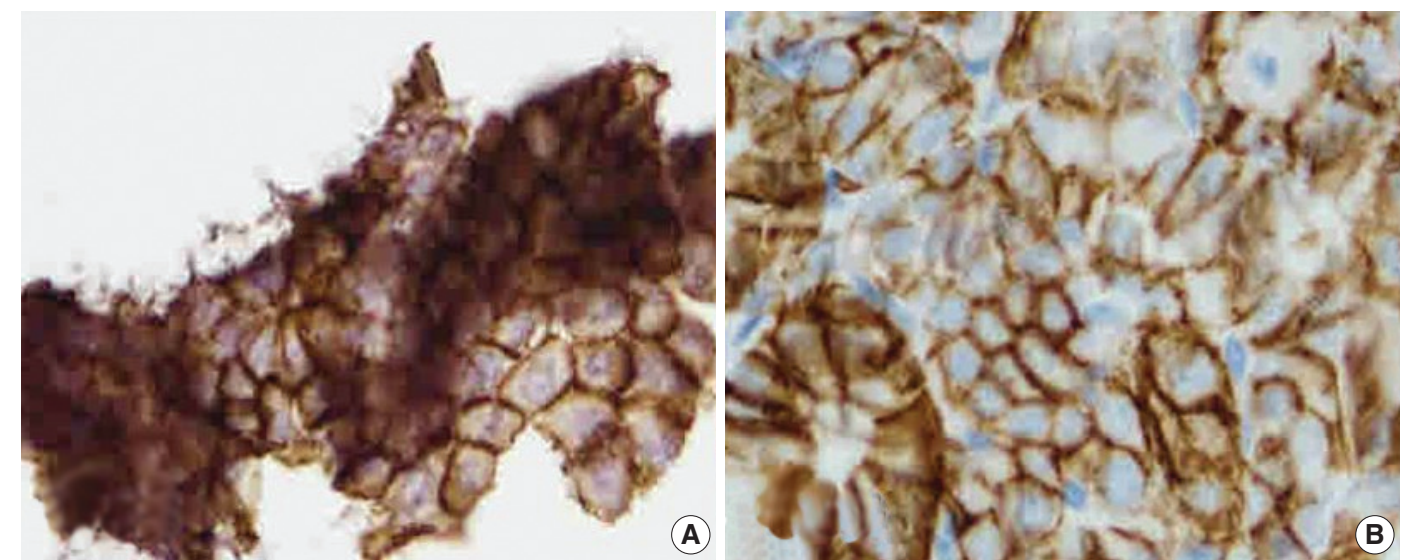

Fig. 4. (A) Cytoplasm-membranous CD56 positivity in a thyroid lesion diagnosed as category III on liquid-based cytology but diagnosed as benign goiter on the histological sample (avidin-biotin-peroxidase complex, × 400). (B) Diffuse cytoplasmic and membranous CD56 positivity on the histological sample for the same case (avidin-biotin-peroxidase complex, $\times 400$ ).

Table 2. Descriptive statistics for each immunocytochemical marker in the cytohistological series

\begin{tabular}{lccccc}
\hline & Sensitivity & Specificity & $\begin{array}{c}\text { Diagnostic } \\
\text { accuracy }\end{array}$ & OR (95\% Cl) & p-value \\
\hline CD56 & 90.0 & 83.3 & 88.4 & $45.0(3.3-604)$ & .004 \\
HBME-1 & 95.0 & 83.3 & 84.6 & $28.3(2.3-336)$ & .008 \\
\hline
\end{tabular}

A p-value less than .05 is considered significant.

$\mathrm{OR}$, odds ratio; $\mathrm{Cl}$, confidence interval; HBME-1, human bone marrow endothelial cell marker-1.

sensitivity (75.0\% vs $90 \%)$ and higher specificity (93.8\% vs $83.3 \%$ ) in detecting malignancy compared to using CD56 alone (Table 3). Also the diagnostic accuracy was $90.0 \%$ in detecting malignancy when compared to using CD 56 or HBME-1 alone.

\section{DISCUSSION}

As we predicted, CD56 showed high sensitivity (90\%) and relatively high diagnostic accuracy in diagnoses in category III thyroid cytology. Therefore, we believe CD56 is a very effective screening marker. CD56 has been recognized as an effective marker in previous studies as well. Many studies showed that CD56 is less prominent in PTC samples. In our study, we showed that CD56 is a useful marker in thyroid cytology, which differs from previous studies in which CD56 was used in thyroid tissue samples.

Recent studies have examined the efficacy of CD56 immunostaining and the role of CD56 when used as a panel for HBME-1 immunostaining and for determination of thyroid FNAC positivity and malignancy. 34,35

Samples showing fewer papillary structures, pseudo-inclusion in the nucleus, focal nuclear pleomorphism, and atypia can be confusing and might lead to a diagnostic dilemma. Any morphological similarity between benign lesions and PTC may be 
Table 3. Staining with a double immunocytochemistry combination of CD56 and HBME-1

\begin{tabular}{lcc}
\hline & \multicolumn{2}{c}{ CD56/HBME-1 $(n=20)$} \\
\cline { 2 - 3 } & CD56-/HBME-1 ${ }^{+}$ & CD56 $^{+} / \mathrm{HBME}^{-1^{-}}$ \\
\hline Benign & $1(5)$ & $3(15)$ \\
Malignant & $15(75)$ & $1(5)$ \\
\hline
\end{tabular}

Values are presented as number (\%).

HBME-1, human bone marrow endothelial cell marker-1.

the cause of misdiagnosis between FNAC and histological surgical specimens. For example, when Hashimoto's thyroiditis has nuclear atypia, empty chromatin, or nuclear groove, this can be confusing and might result in misdiagnosis. ${ }^{26}$

The relatively low specificity of FNAC can be further improved by applying an ancillary technique (e.g., immunocytochemistry and molecular marker). For this reason, effective dye markers (HBME-1, galatin-3) are attracting attention. ${ }^{35,37}$

HBME-1 displayed high sensitivity and high specificity in detecting PTC in many cases. ${ }^{34}$ Additional reports suggest that mixed panels of immunostaining markers would provide more accurate diagnoses. ${ }^{12-16,20,22,25,26,33,36}$

Many studies were aimed at finding a sole maker for identifying malignancy accurately. CD56 was one of the most preferred markers for thyroid epithelial neoplasm in an immunohistochemistry panel. ${ }^{21-26,37}$ While the exact mechanism is not well known, CD56 is noted in multiple sites (e.g., neuron, mesenchymal tissue, and endocrine cells). ${ }^{21-26}$ Some studies correlated different CD 56 expression with tumor cell migrations. ${ }^{29}$ In previous studies of thyroid histological samples, CD56 was seen as a promising immunostaining marker expressed in most normal thyroid tissues including goiter, Grave disease, and Hashimoto thyroiditis. CD56 showed a negative staining pattern in PTC tissues including variants of PTC. ${ }^{27,29,34,40-42}$ Indeed, in one study, the low expression of CD56 in PTC was shown to be highly specific in both single-use and dyed panel applications. 29,34

Although the data of El Demellawy et al..$^{40}$ showed that CD56 was expressed in all benign lesions, our study showed slightly less (83\%) positive expression of CD56 in benign lesions. Interestingly, all but one malignant lesion showed negative CD 56 expression. We also compared CD56 with HBME-1 because HBME-1 is a preferred marker in building an immunocytochemistry panel, which could improve diagnostic accuracy. Our study is the first we know of that reveals the diagnostic usefulness of CD56 immunostaining for Bethesda’s category III samples using thyroid cytology.

CD56 is usually studied in formalin-fixed and paraffin-embedded material. ${ }^{29,34,41,42}$ We demonstrated the usefulness of immu- nodiffusion with cells that are thought of as atypical when using the cell transfer method. The positive features of FNAC are cost effectiveness, time saving, and practicality; also, the test is not invasive. ${ }^{35}$ An ancillary technique such as immunocytochemistry or molecular testing can add cost but can also save money in the end by avoiding unnecessary thyroidectomy or lifelong drug treatment.

One limitation of our study was the relatively small sample size. Further study conducted with a larger number of samples should bring about more definitive conclusions.

Instead of using the well-known cell-block technique, we immunostained LBC for two reasons. First, LBC showed reliable results in immunostaining. Second, fixation can cause the cellblock to show false positive or false-negative, a problem we did not encounter while using LBC immunostaining. ${ }^{34,35}$

Our preliminary results show that CD56 is likely to be a very effective and reliable marker for ruling out PTC. We also suggest that $\mathrm{CD} 56$ be used in FNA when it is difficult to confirm the diagnosis using HBME-1 alone. Also, its efficacy can be enhanced through combination with other immunostaining markers.

\section{ORCID}

Hyunseo Cha: https://orcid.org/0000-0003-0243-9589

Ju Yeon Pyo: https://orcid.org/0000-0002-9198-5065

Soon Won Hong: https://orcid.org/0000-0002-0324-2414

\section{Conflicts of Interest}

No potential conflict of interest relevant to this article was reported.

\section{REFERENCES}

1. American Thyroid Association (ATA) Guidelines Taskforce on Thyroid Nodules and Differentiated Thyroid Cancer, Cooper DS, Doherty GM, et al. Revised American Thyroid Association management guidelines for patients with thyroid nodules and differentiated thyroid cancer. Thyroid 2009; 19: 1167-214.

2. Ahn HS, Kim HJ, Kim KH, et al. Thyroid cancer screening in South Korea increases detection of papillary cancers with no impact on other subtypes or thyroid cancer mortality. Thyroid 2016; 26: 1535-40.

3. Cha YJ, Pyo JY, Hong S, et al. Thyroid fine-needle aspiration cytology practice in Korea. J Pathol Transl Med 2017; 51: 521-7.

4. Powsner SM, Costa J, Homer RJ. Clinicians are from Mars and pathologists are from Venus. Arch Pathol Lab Med 2000; 124: 1040-6.

5. Cibas ES, Ali SZ; NCI Thyroid FNA State of the Science Conference. 
The Bethesda System For Reporting Thyroid Cytopathology. Am J Clin Pathol 2009; 132: 658-65.

6. Cibas ES, Ali SZ. The 2017 Bethesda System for Reporting Thyroid Cytopathology. Thyroid 2017; 27: 1341-6.

7. Jung CK, Hong S, Bychkov A, Kakudo K. The use of fine-needle aspiration (FNA) cytology in patients with thyroid nodules in Asia: a brief overview of studies from the Working Group of Asian Thyroid FNA Cytology. J Pathol Transl Med 2017; 51: 571-8.

8. Kang Y, Lee YJ, Jung J, Lee Y, Won NH, Chae YS. Morphometric analysis of thyroid follicular cells with atypia of undetermined significance. J Pathol Transl Med 2016; 50: 287-93.

9. Yoo C, Choi HJ, Im S, et al. Fine needle aspiration cytology of thyroid follicular neoplasm: cytohistologic correlation and accuracy. Korean J Pathol 2013; 47: 61-6.

10. Oosthuizen JL, Walker B, Todorovic E, Masoudi H, Wiseman SM. The presence of papillary features in thyroid nodules diagnosed as atypia of undetermined significance or follicular lesion of undetermined significance increases cancer risk and should influence treatment. Am J Surg 2018; 215: 819-23.

11. Yashaswini R, Suresh TN, Sagayaraj A. Cytological evaluation of thyroid lesions by nuclear morphology and nuclear morphometry. J Cytol 2017; 34: 197-202.

12. Vivero M, Renshaw AA, Krane JF. Adequacy criteria for thyroid FNA evaluated by ThinPrep slides only. Cancer Cytopathol 2017; 125: 534-43.

13. Rossi M, Lupo S, Rossi R, et al. Proposal for a novel management of indeterminate thyroid nodules on the basis of cytopathological subclasses. Endocrine 2017; 57: 98-107.

14. Satoh S, Yamashita H, Kakudo K. Thyroid cytology: The Japanese system and experience at Yamashita Thyroid Hospital. J Pathol Transl Med 2017; 51: 548-54.

15. Kim SJ, Roh J, Baek JH, et al. Risk of malignancy according to subclassification of the atypia of undetermined significance or follicular lesion of undetermined significance (AUS/FLUS) category in the Bethesda system for reporting thyroid cytopathology. Cytopathology 2017; 28: 65-73.

16. Misiakos EP, Margari N, Meristoudis C, et al. Cytopathologic diagnosis of fine needle aspiration biopsies of thyroid nodules. World J Clin Cases 2016; 4: 38-48.

17. Kholová I, Ludvíková M. Thyroid atypia of undetermined significance or follicular lesion of undetermined significance: an indispensable Bethesda 2010 diagnostic category or waste garbage? Acta Cytol 2014; 58: 319-29.

18. Shi $Y$, Ding X, Klein M, et al. Thyroid fine-needle aspiration with atypia of undetermined significance: a necessary or optional category? Cancer 2009; 117: 298-304.
19. Garg S, Naik LP, Kothari KS, Fernandes GC, Agnihotri MA, Gokhale JC. Evaluation of thyroid nodules classified as Bethesda category III on FNAC. J Cytol 2017; 34: 5-9.

20. Rossi ED, Martini M, Capodimonti S, et al. Diagnostic and prognostic value of immunocytochemistry and $B R A F$ mutation analysis on liquid-based biopsies of thyroid neoplasms suspicious for carcinoma. Eur J Endocrinol 2013; 168: 853-9.

21. Fadda G, Rossi ED, Raffaelli M, et al. Follicular thyroid neoplasms can be classified as low- and high-risk according to HBME-1 and Galectin-3 expression on liquid-based fine-needle cytology. Eur J Endocrinol 2011; 165: 447-53.

22. Ohori NP, Nikiforova MN, Schoedel KE, et al. Contribution of molecular testing to thyroid fine-needle aspiration cytology of "follicular lesion of undetermined significance/atypia of undetermined significance". Cancer Cytopathol 2010; 118: 17-23.

23. Cochand-Priollet B, Dahan H, Laloi-Michelin M, et al. Immunocytochemistry with cytokeratin 19 and anti-human mesothelial cell antibody (HBME1) increases the diagnostic accuracy of thyroid fineneedle aspirations: preliminary report of 150 liquid-based fine-needle aspirations with histological control. Thyroid 2011; 21: 1067-73.

24. Abouhashem NS, Talaat SM. Diagnostic utility of CK19 and CD56 in the differentiation of thyroid papillary carcinoma from its mimics. Pathol Res Pract 2017; 213: 509-17.

25. Solmaz OA. Diagnostic importance of CD56 with fine-needle aspiration cytology in suspected papillary thyroid carcinoma cases. Cytojournal 2018; 15:3.

26. Mokhtari M, Eftekhari M, Tahririan R. Absent CD56 expression in papillary thyroid carcinoma: A finding of potential diagnostic value in problematic cases of thyroid pathology. J Res Med Sci 2013; 18: 1046-50.

27. Nechifor-Boila A, Borda A, Sassolas G, et al. Immunohistochemical markers in the diagnosis of papillary thyroid carcinomas: the promising role of combined immunostaining using HBME-1 and CD56. Pathol Res Pract 2013; 209: 585-92.

28. Nechifor-Boilă A, Cătană R, Loghin A, Radu TG, Borda A. Diagnostic value of HBME-1, CD56, galectin-3 and cytokeratin-19 in papillary thyroid carcinomas and thyroid tumors of uncertain malignant potential. Rom J Morphol Embryol 2014; 55: 49-56.

29. Scarpino S, Di Napoli A, Melotti F, Talerico C, Cancrini A, Ruco L. Papillary carcinoma of the thyroid: low expression of NCAM (CD56) is associated with downregulation of VEGF-D production by tumour cells. J Pathol 2007; 212: 411-9.

30. Shahebrahimi K, Madani SH, Fazaeli AR, Khazaei S, Kanani M, Keshavarz A. Diagnostic value of CD56 and nm23 markers in papillary thyroid carcinoma. Indian J Pathol Microbiol 2013; 56: 2-5.

31. Huang L, Wang X, Huang X, et al. Diagnostic significance of CK19, 
galectin-3, CD56, TPO and Ki67 expression and BRAF mutation in papillary thyroid carcinoma. Oncol Lett 2018; 15: 4269-77.

32. Muzafar A, Bukhari MH, Qureshi IU. A study of galactin-3 on fine needle aspiration as a diagnostic marker differentiating benign from malignant thyroid neoplasm. Pak J Med Sci 2017; 33: 726-31.

33. Golu I, Vlad MM, Dema A, et al. The absence of CD56 expression can differentiate papillary thyroid carcinoma from other thyroid lesions. Indian J Pathol Microbiol 2017; 60: 161-6.

34. Abd El Atti RM, Shash LS. Potential diagnostic utility of CD56 and claudin- 1 in papillary thyroid carcinoma and solitary follicular thyroid nodules. J Egypt Natl Canc Inst 2012; 24: 175-84.

35. Ceyran AB, Şenol S, Şimşek BÇ, Sağıroğlu J, Aydın A. Role of cd56 and e-cadherin expression in the differential diagnosis of papillary thyroid carcinoma and suspected follicular-patterned lesions of the thyroid: the prognostic importance of e-cadherin. Int J Clin Exp Pathol 2015; 8: 3670-80.

36. Erdogan-Durmus S, Ozcan D, Yarikkaya E, Kurt A, Arslan A. CD56, HBME-1 and cytokeratin 19 expressions in papillary thyroid carcinoma and nodular thyroid lesions. J Res Med Sci 2016; 21: 49.

37. Oh EJ, Hong SW, Jeong HJ, Yoon SO. The diagnostic approach to fine-needle aspiration of malignant lymphoma: using cytomorphology and immunocytochemistry with cell transfer method. Diagn Cytopathol 2014; 42: 671-9.

38. Sherman ME, Jimenez-Joseph D, Gangi MD, Rojas-Corona RR. Immunostaining of small cytologic specimens: facilitation with cell transfer. Acta Cytol 1994; 38: 18-22.

39. Zu Y, Gangi MD, Yang GC. Ultrafast Papanicolaou stain and celltransfer technique enhance cytologic diagnosis of Hodgkin lymphoma. Diagn Cytopathol 2002; 27: 308-11.

40. El Demellawy D, Nasr AL, Babay S, Alowami S. Diagnostic utility of CD56 immunohistochemistry in papillary carcinoma of the thyroid. Pathol Res Pract 2009; 205: 303-9.

41. Zeromski J, Bagnasco M, Paolieri F, Dworacki G. Expression of CD56 (NKH-1) differentiation antigen in human thyroid epithelium. Clin Exp Immunol 1992; 89: 474-8.

42. Ozolins A, Narbuts Z, Strumfa I, Volanska G, Stepanovs K, Gardovskis J. Immunohistochemical expression of HBME-1, E-cadherin, and CD56 in the differential diagnosis of thyroid nodules. Medicina (Kaunas) 2012; 48: 507-14. 